\title{
Protective effects of patchouli alcohol isolated from Pogostemon cablin on lipopolysaccharide-induced acute lung injury in mice
}

\author{
ZUQING SU ${ }^{1,2^{*}}$, JINBIN LIAO $^{1,3^{*}}$, YUHONG LIU $^{1 *}$, YONGZHUO LIANG $^{1}$, HAIMING CHEN $^{1}$, \\ XIAOYING $\mathrm{CHEN}^{1}$, XIAOPING LAI ${ }^{1,4}$, XUEXUAN FENG ${ }^{1}$, DIANWEI WU ${ }^{5}$, \\ YIFENG ZHENG $^{1}$, XIAOJUN ZHANG ${ }^{1}$ and YUCUI LI ${ }^{1}$
}

\begin{abstract}
${ }^{1}$ School of Chinese Materia Medica, Guangzhou University of Chinese Medicine, Guangzhou, Guangdong 510006; ${ }^{2}$ Guangdong Provincal Hospital of Chinese Medicine, Guangzhou, Guangdong 510120;

${ }^{3}$ Pharmaceutical Department, Guangdong Second Province Hospital of Traditional Chinese Medicine, Guangzhou, Guangdong 510095; ${ }^{4}$ Dongguan Mathematical Engineering Academy of Chinese Medicine, Guangzhou University of Chinese Medicine, Dongguan, Guangdong 523808; ${ }^{5}$ Department of Pharmacy, Shantou Hospital of Traditional Chinese Medicine, Shantou, Guangdong 515031, P.R. China
\end{abstract}

Received October 30, 2014; Accepted October 22, 2015

DOI: $10.3892 / e t m .2015 .2918$

\begin{abstract}
Patchouli alcohol (PA) is a tricyclic sesquiterpene isolated from Pogostemon cablin, which exerts anti-inflammatory, anti-influenza and cognitive-enhancing bioactivities. The present study aimed to investigate the protective effects of PA on acute lung injury (ALI) induced by intratracheal instillation of lipopolysaccharide (LPS) in mice. Dexamethasone was used as a positive drug for protection against LPS-induced ALI. The results of the present study demonstrated that pretreatment with PA significantly increased survival rate, attenuated histopathologic damage and lung edema, and decreased the protein content in the bronchoalveolar lavage fluid (BALF) of mice with ALI. Furthermore, PA significantly inhibited the expression levels of proinflammatory cytokines, including tumor necrosis factor (TNF)- $\alpha$ and interleukin (IL)-6 in the BALF, downregulated the levels of myeloperoxidase and malondialdehyde, and upregulated the activity levels of superoxide dismutase and glutathione peroxidase in lung tissue. These results indicated that PA may exert potent protective effects against LPS-induced ALI in
\end{abstract}

Correspondence to: Dr Yucui Li or Dr Xiaojun Zhang, School of Chinese Materia Medica, Guangzhou University of Chinese Medicine, 232 Waihuan Dong Road, Guangzhou, Guangdong 510006, P.R. China

E-mail: liyucui@gzucm.edu.cn

E-mail: zhangxj@gzucm.edu.cn

*Contributed equally

Key words: patchouli alcohol, acute lung injury, lipopolysaccharide, anti-inflammatory activity, antioxidative activity mice, the mechanisms of which are possibly associated with the anti-inflammatory and antioxidative activities of PA.

\section{Introduction}

Acute lung injury (ALI) is defined as a complex syndrome associated with an intense pulmonary inflammation (1). Clinically, ALI is characterized by lung edema, neutrophil infiltration, hemorrhage, bronchiole epithelial desquamation, and marked thickening of the alveolar wall $(2,3)$. Severe ALI may lead to enhanced permeability and pulmonary edema, acute respiratory distress syndrome, and eventual respiratory failure $(4,5)$. Despite marked advances in the treatment of ALI in recent years, ALI remains a life-threatening disease with a high mortality rate of $30-40 \%$ (6). Therefore, it is crucial that novel effective therapeutic strategies for the treatment of ALI are developed.

Lipopolysaccharide (LPS) is the major constituent of the outer membrane of Gram-negative bacteria, and is composed of a polar lipid head group and a chain of repeating disaccharides. LPS has been demonstrated to have an important role in the pathogenesis of ALI (7-9). In vivo intratracheal instillation of LPS, which causes pulmonary inflammation without inducing systemic inflammation and multi-organ failure, has been widely accepted as an ideal pharmacological tool for the in vivo induction of ALI in model systems $(10,11)$. LPS-induced ALI leads to an inflammatory response cascade, characterized by the release of various proinflammatory mediators (12). Activated macrophages release a broad spectrum of cytokines and inflammatory mediators, including tumor necrosis factor (TNF)- $\alpha$ and interleukin (IL)-6, which not only promote inflammatory injury, but also induce neutrophil influx into the lung parenchyma (13). Neutrophil activation induces the excessive production of reactive oxygen species (ROS) and the release of granular enzymes, including myelo- 
peroxidase (MPO), which was associated with ALI in previous studies $(14,15)$. Furthermore, excessive ROS production may induce impairment of DNA and membrane lipid damage, leading to lipid peroxidation and the associated production of malondialdehyde (MDA), which is a cell destruction-dependent index of oxidative injury (16). Tissues are protected from ROS-induced toxic damage by antioxidative enzymes, such as superoxide dismutase (SOD) and glutathione peroxidase (GPx) (17).

Pogostemon cablin (Blanco) Benth. (Lamiaceae) is traditionally used in China to treat various illnesses, including fever, common cold, diarrhea and nausea (18-20). Previous studies have demonstrated that $P$. cablin also exerts numerous bioactivities, including radical-scavenging, anti-microbial, analgesic and anti-inflammatory activities (21-23). Patchouli alcohol (PA; Fig. 1), which is a tricyclic sesquiterpene, is the main active ingredient of $P$. cablin (24). The authors of the present study have previously demonstrated that PA inhibits LPS-induced inflammatory responses in LPS-stimulated RAW264.7 macrophages, and also possesses potent anti-inflammatory activity in animal models of inflammation $(20,24)$. Furthermore, oral administration of PA has been demonstrated to offer protection against influenza virus infection in mice via the enhancement of host immune responses, and the attenuation of systemic and pulmonary inflammatory responses (18). In addition, it has been reported that pretreatment with PA attenuates ROS generation following $A \beta_{25-35}$-induced toxicity (25). These findings indicated that PA possesses anti-inflammatory and antioxidative activities. However, to the best of our knowledge, the present study is the first to report the effects of PA on LPS-induced ALI in a murine model. Whether PA exerts protective effects on LPS-induced ALI in mice remains unclear. Therefore, the aim of the present study was to investigate these protective effects and the possible mechanism offered by PA against LPS-induced ALI in mice.

\section{Materials and methods}

Plants. The aerial parts of $P$. cablin were obtained from Guangzhou Zhixing Pharmaceutical Co., Ltd. (Guangzhou, China) and authenticated by Professor Lai Xiaoping, an experienced pharmacognosist, at the School of Chinese Materia Medica, Guangzhou University of Chinese Medicine (Guangzhou, China). The voucher specimen was deposited in the herbarium of the School of Chinese Materia Medica, Guangzhou University of Chinese Medicine.

Isolation and purification of PA. PA was isolated from $P$. cablin according to methods described in our previous studies $(20,24,26)$. The aerial parts of $P$. cablin, weighing $18 \mathrm{~kg}$, were refluxed with $95 \% \mathrm{v} / \mathrm{v}$ ethanol/aqueous (40 liters $\mathrm{x} 2 ; 60 \mathrm{~min}$ each time) and the extract was evaporated under a vacuum in order to obtain a residue. The residue was subsequently dissolved in acetone and subjected to column chromatography over silica gel eluted with a petroleum ether/ethyl acetate/0.1\% formic acid (20:1:0.1, 9:1:0.1, 8:3:0.1 and 7:4:0.1, vol/vol/vol) gradient elution system with increasing polarity, in order to produce a series of fractions. Thin layer chromatography was performed to distinguish the resulting fractions, and the fraction eluted with petroleum ether/ethyl acetate/0.1\% formic acid (9:1:0.1) was combined and subsequently evaporated to yield a yellowish oily liquid. White crystals of PA were obtained following crystallization from n-hexane. The purity of PA was analyzed using analytical gas chromatography (GC; Xcalibur 3.0; Thermo Fisher Scientific, Inc., Waltham, MA, USA) and the chemical structure was confirmed by Fourier transform infrared spectroscopy (IRSolution 1.4; Thermo Fisher Scientific, Inc.), mass spectrometry (Xcalibur 3.0) and nuclear magnetic resonance spectroscopy (Topsin 3.2; Bruker BioSpin, Zurich, Switzerland). GC analysis demonstrated the purity of PA was $>98 \%$ (26).

Animals. Male Kunming (KM) mice, 4-5 weeks old and weighing 20-22 g, were obtained from the Guangdong Provincial Medical Animal Experimental Center (certificate no. SCXK2013-0002; Foshan, China). The mice were maintained in microisolator cages with a regular temperature $\left(24 \pm 1^{\circ} \mathrm{C}\right)$, relative humidity $(55 \pm 10 \%)$ and a 12 -h light/dark cycle. All the experimental protocols and schedules involving animals were approved by the Animal Welfare Committee of Guangzhou University of Chinese Medicine.

Reagents. Dexamethasone (DEX) was purchased from Guangdong Huanan Pharmaceutical Group Co., Ltd. (Dongguan, China). LPS (Escherichia coli O111:B4) was purchased from Sigma-Aldrich (St. Louis, MO, USA). TNF- $\alpha$ and IL-6 enzyme-linked immunosorbent assay kits were obtained from eBioscience, Inc., (San Diego, CA, USA). The MPO, GPx, MDA, SOD and Coomassie (Bradford) Protein Assay kits were purchased from the Nanjing Jiancheng Bioengineering Institute (Nanjing, China).

Survival studies. For the analysis of mortality rate, 100 mice were randomly divided into five groups $(n=20)$ : Sham; LPS; and PA $(10,20$ and $40 \mathrm{mg} / \mathrm{kg})$ groups. Mice in the PA groups were intragastrically administered 10, 20 and $40 \mathrm{mg} / \mathrm{kg}$ PA, whereas the sham and LPS groups were administered $1 \%$ poloxamer 407 once a day for 7 consecutive days. The mice were anesthetized using 3\% chloral hydrate (Aladdin Reagent Co., Ltd., Shanghai, China) $1 \mathrm{~h}$ after the final administration. Mice in the LPS and PA groups were administered $20 \mathrm{mg} / \mathrm{kg}$ LPS via intratracheal instillation, whereas mice in the sham group was administered an equal volume of phosphate-buffered saline (PBS). The mortality rate was recorded for 5 days.

Murine model of LPS-induced ALI. A total of 168 mice were randomly divided into six groups: Sham, LPS, $5 \mathrm{mg} / \mathrm{kg} \mathrm{DEX}$, and 10, 20 and $40 \mathrm{mg} / \mathrm{kg}$ PA groups. Mice from the sham and LPS groups were administered $1 \%$ poloxamer 407 , whereas the PA groups were administered 10,20 or $40 \mathrm{mg} / \mathrm{kg}$ PA daily for 7 consecutive days. The DEX group was administered $5 \mathrm{mg} / \mathrm{kg}$ DEX daily for 5 consecutive days. The mice were anesthetized $1 \mathrm{~h}$ after the final administration. The LPS, PA and DEX groups were administered $5 \mathrm{mg} / \mathrm{kg}$ LPS by intratracheal instillation, whereas the sham group was administered an equal volume of PBS. All mice were sacrificed by cervial dislocation after $24 \mathrm{~h}$, and lung tissue and bronchoalveolar lavage fluid (BALF) samples were harvested for further study. 
Measurement of protein content in BALF. The mice lungs were lavaged with $1.5 \mathrm{ml}$ PBS three times and $1.35 \mathrm{ml}$ BALF was recovered with $\sim 90 \pm 2 \%$ recovery rates. The BALF samples were centrifuged at $800 \mathrm{x}$ g for $10 \mathrm{~min}$ at $4^{\circ} \mathrm{C}$ and the supernatants were collected in order to measure the protein content using Coomassie (Bradford) Protein Assay kits (Thermo Fisher Scientific, Inc.).

Measurement of lung edema. The lung wet/dry weight (W/D) ratios were determined to evaluate the protective effects of PA on LPS-induced lung edema. Upon completion of the experiments, lung tissues were excised and immediately weighed to record the 'wet' weight, to obtain the 'dry' weight the tissues were weighed after being heated at $80^{\circ} \mathrm{C}$ for $48 \mathrm{~h}$.

Histopathologic examination. Lung tissues were fixed in $4 \%$ paraformaldehyde, embedded in paraffin and cut into $4 \mu \mathrm{m}$ sections. The sections were subsequently stained with hematoxylin and eosin according to the manufacturer's protocol (Nanjing Jiancheng Bioengineering Institute), examined, and images were captured using a TE2000-S inverted microscope (Nikon Corporation, Tokyo, Japan).

Measurement of MPO, SOD, GPx and MDA. Lung tissues were homogenized using PBS and centrifuged at 14,167 x g for $10 \mathrm{~min}$ at $4^{\circ} \mathrm{C}$. Subsequently, the supernatants were collected and the levels of MPO, SOD, GPx and MDA in the lung tissue were examined by the respective assay kits (Nanjing Jiancheng Bioengineering Institute), according to the manufacturer's protocol.

Measurement of proinflammatory cytokines in BALF. The expression levels of the proinflammatory cytokines TNF- $\alpha$ and IL- 6 were examined in the BALF using enzyme-linked immunosorbent assay kits (eBioscience, CA, USA), according to the manufacturer's protocols.

Statistical analysis. Data are expressed as the mean \pm standard error of the mean. Experimental values were analyzed by one-way analysis of variance using SPSS 17.0 statistical analysis software (SPSS, Inc., Chicago, IL, USA). Mortality was presented as Kaplan-Meier curves and differences were assessed by the log-rank test. $\mathrm{P}<0.05$ was considered to indicate a statistically significant difference.

\section{Results}

PA improved the survival rate of LPS-induced ALI in mice. As compared with the sham group, which had a survival rate of $100 \%$, the survival rate of the LPS group was significantly decreased (20\%; $\mathrm{P}<0.01)$ (Fig. 2). The 5-day survival rates of the 10,20 and $40 \mathrm{mg} / \mathrm{kg}$ PA pretreatment groups were 35 , 40 and $55 \%(\mathrm{P}<0.05$ vs. the LPS group), respectively. The Kaplan-Meier survival analysis demonstrated that pretreatment with PA protected mice with ALI from mortality in a dose-dependent manner.

Effects of PA on the protein content in BALF. Protein content in the BALF corresponds to the vascular permeability of the lungs. The BALF protein content in the LPS group was

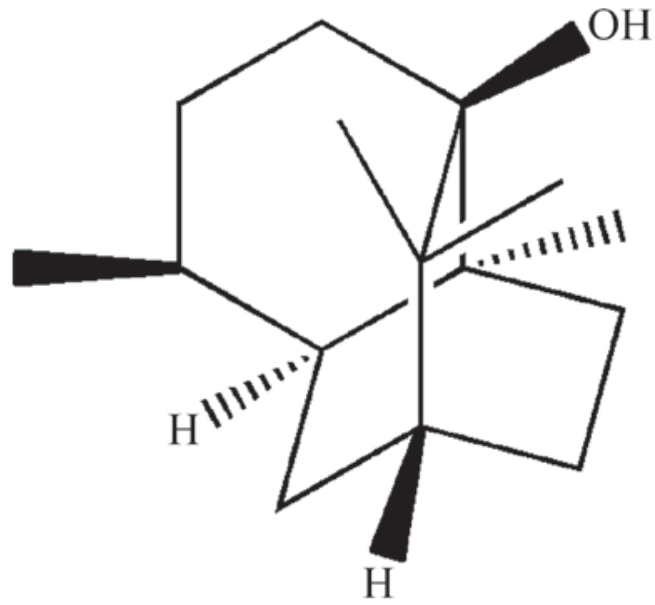

Figure 1. Structure of patchouli alcohol.

significantly increased $(\mathrm{P}<0.01)$, as compared with the sham group (Fig. 3A). Conversely, the BALF protein content was significantly decreased in the 20 and $40 \mathrm{mg} / \mathrm{kg}$ PA $(\mathrm{P}<0.05)$ and $5 \mathrm{mg} / \mathrm{kg}$ DEX $(\mathrm{P}<0.01)$ groups, as compared with the LPS group. These results suggest that PA may improve LPS-induced pulmonary vascular permeability in mice.

Effects of PA on lung edema. The W/D ratios of the mice with ALI were evaluated, in order to assess the severity of pulmonary edema. The lung W/D weight ratio significantly increased $(\mathrm{P}<0.01)$ following LPS stimulation, as compared with the sham group (Fig. 3B). However, pretreatment with 20 and $40 \mathrm{mg} / \mathrm{kg}$ PA and $5 \mathrm{mg} / \mathrm{kg}$ DEX significantly suppressed $(\mathrm{P}<0.05)$ lung W/D weight ratio, as compared with the LPS group.

Effects of PA on histopathologic alterations. In the sham group, the structure of the alveolar wall was normal and minimal inflammation was detected (Fig. 4). Characteristic histopathologic alterations in the lung tissue were observed following LPS challenge, including lung edema, neutrophil infiltration, hemorrhage and marked thickening of the alveolar wall. Pretreatment with 20 and $40 \mathrm{mg} / \mathrm{kg}$ PA and $5 \mathrm{mg} / \mathrm{kg}$ DEX markedly attenuated these histopathologic changes.

Effects of PA on MPO, MDA, SOD and GPx levels. In the LPS group, a significant increase in MPO (Fig. 5A) activity and MDA levels (Fig. 5B), and a significant decrease $(\mathrm{P}<0.01)$ in SOD (Fig. 5C) and GPx (Fig. 5D) activities were detected in the pulmonary homogenate, as compared with the sham group. However, the mice in the groups pre-treated with PA $(10,20$ and $40 \mathrm{mg} / \mathrm{kg})$ or DEX $(5 \mathrm{mg} / \mathrm{kg})$ demonstrated significantly decreased MDA levels $(\mathrm{P}<0.05)$, as compared with the LPS group (Fig. 5B). Furthermore, treatment with 20 and $40 \mathrm{mg} / \mathrm{kg}$ PA and DEX significantly inhibited the activities of MPO (Fig. 5A), and increased SOD (Fig. 5C) and GPx (Fig. 5D) activities in the pulmonary homogenate of mice with ALI $(\mathrm{P}<0.05)$, as compared with the LPS group.

Effects of PA on the expression of inflammatory cytokines in BALF. The expression levels of TNF- $\alpha$ (Fig. 6A) and 


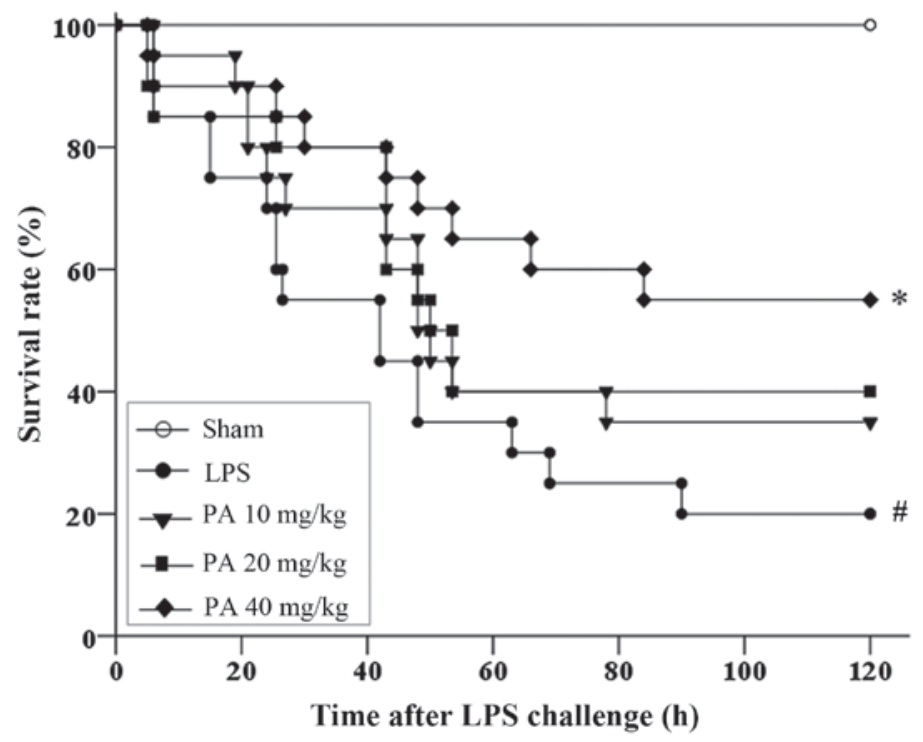

Figure 2. Effects of patchouli alcohol (PA) on lipopolysaccharide (LPS)-induced mortality in mice ( $\mathrm{n}=10)$. The mortality rates of all the groups were recorded for 5 days. Data are presented as Kaplan-Meier curves and comparisons were made using the log rank test. ${ }^{*} \mathrm{P}<0.05$ vs. the LPS group; ${ }^{*} \mathrm{P}<0.01$ vs. the sham group.
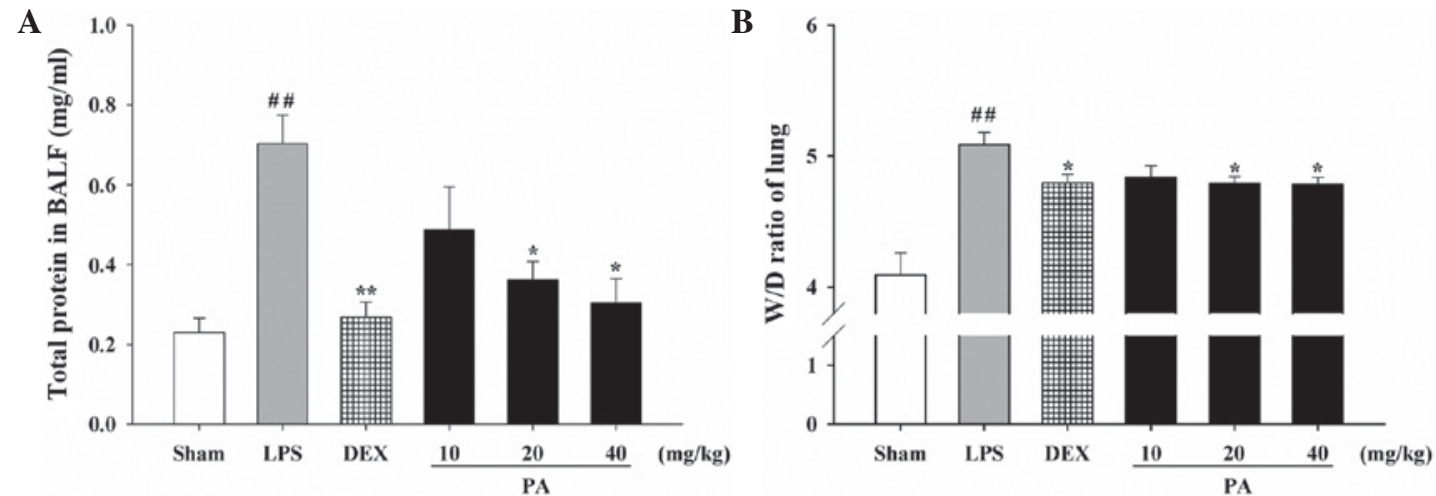

Figure 3. Effects of patchouli alcohol (PA) on total protein concentration in bronchoalveolar lavage fluid (BALF) and lung edema. Mice were administered 10, 20 and $40 \mathrm{mg} / \mathrm{kg}$ PA, $5 \mathrm{mg} / \mathrm{kg}$ dexamethasone (DEX) or an equal volume of vehicle. Mice in the lipopolysaccharide (LPS), PA and DEX groups were administered $5 \mathrm{mg} / \mathrm{kg}$ LPS, whereas mice from the sham group received an equal volume of phosphate-buffered saline. Following $24 \mathrm{~h}$, the mice were sacrificed by cervical dislocation, and subsequently, the BALF and lung tissue samples were harvested for the analysis of $(A)$ total protein concentration $(n=8)$ and $(B)$ lung edema $(n=6)$. Data are presented as the mean \pm standard error of the mean. ${ }^{\# / t} \mathrm{P}<0.01$ vs. the sham group; ${ }^{*} \mathrm{P}<0.05$ and ${ }^{* * *} \mathrm{P}<0.01$ vs. the LPS group. W/D, wet/dry ratio.

IL-6 (Fig. 6B) proinflammatory cytokines were significantly increased in the BALF of the LPS group $(\mathrm{P}<0.01)$, as compared with the sham group. Conversely, the expression levels of TNF- $\alpha$ and IL- 6 in the BALF of the 20 and $40 \mathrm{mg} / \mathrm{kg}$ PA and $5 \mathrm{mg} / \mathrm{kg}$ DEX groups were significantly suppressed $(\mathrm{P}<0.05)$, as compared with the LPS group.

\section{Discussion}

Numerous plant-derived natural products, including polyphenols, chlorogenic acid, ethyl gallate, rutin, magnolol and shikonin (10,13,27-29); saponins, esculentoside A, ruscogenin and diosgenin (30-32); and alkaloids, isotetrandrine, oxymatrine and matrine (33-35), have been demonstrated to exert anti-inflammatory effects in animal models of ALI. However, few studies have investigated the protective actions of terpenes on ALI. PA is a tricyclic sesquiterpene hydrocarbon, which has an oral median lethal dose value of $4,693 \mathrm{mg} / \mathrm{kg}$ in mice (20). The present study demonstrated that pretreatment with PA improved the survival rate of mice with LPS-induced ALI. In addition, the beneficial effects of PA pretreatment included attenuation of lung pathological alterations, reduced W/D ratio and protein leakage, reduced elevated proinflammatory cytokine expression, suppression of lipid peroxidation and MPO levels, and enhanced antioxidant enzyme activities. These results indicated that PA may effectively prevent LPS-induced ALI.

Pulmonary edema, which is a typical symptom of ALI, is usually assessed by measuring the W/D ratio (36). Pulmonary edema is characterized by diffuse alveolar damage, marked increases in the permeability of the alveolar-capillary membrane, and accumulation of protein-rich fluid in the interstitial spaces and alveoli (37). In order to quantify the severity of pulmonary edema in the present study, the lung W/D ratio was examined. Pretreatment with PA significantly decreased the lung W/D ratio induced by the LPS challenge. As another index of ALI following LPS exposure, the total protein concentra- 
A

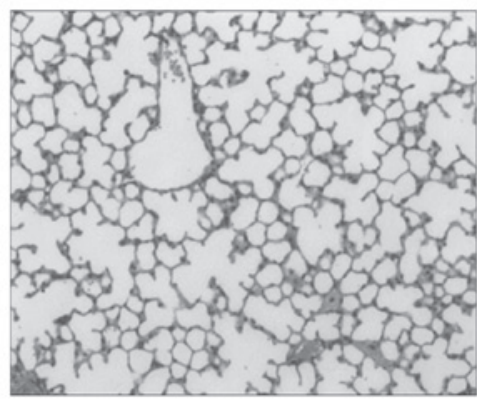

D

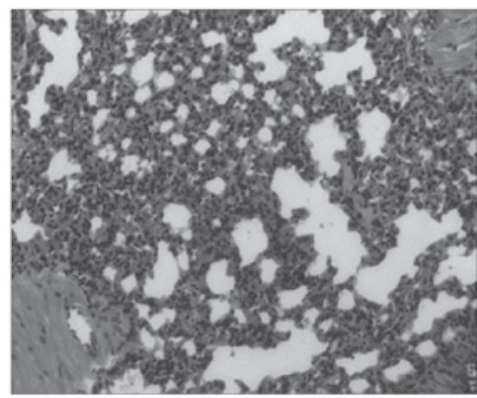

B

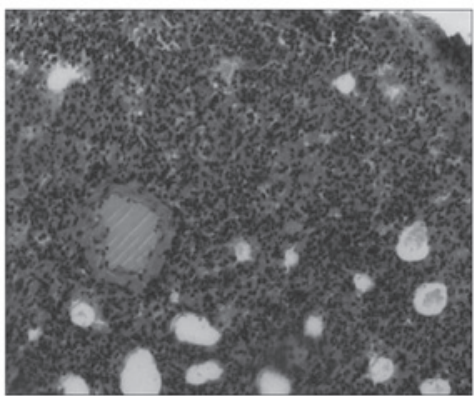

$\mathbf{E}$

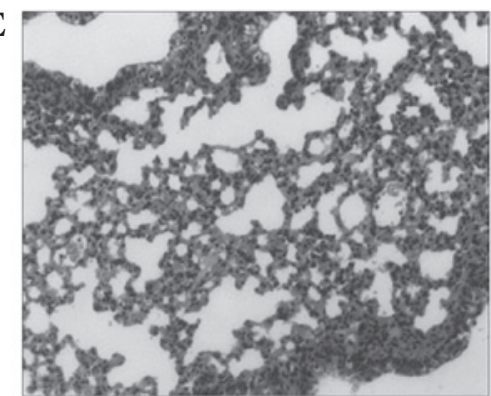

C

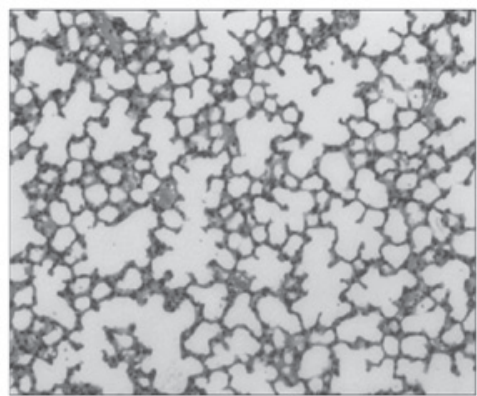

$\mathbf{F}$

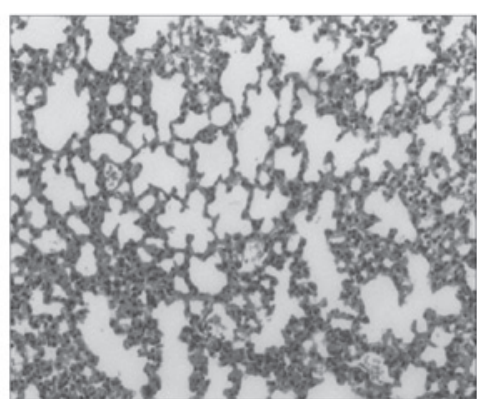

Figure 4. Effects of patchouli alcohol (PA) on histopathologic alterations in mice treated with lipopolysaccharide (LPS). Mice were administered 10, 20 and $40 \mathrm{mg} / \mathrm{kg}$ PA, $5 \mathrm{mg} / \mathrm{kg}$ dexamethasone (DEX) or an equal volume of vehicle. Mice from the LPS, PA and DEX groups were administered $5 \mathrm{mg} / \mathrm{kg}$ LPS, whereas mice in the sham group received an equal volume of phosphate-buffered saline. Following $24 \mathrm{~h}$, the mice were sacrificed by cervical dislocation and the lung tissues were subsequently harvested for histological examination. (A) Sham group; (B) LPS group; (C) LPS + $5 \mathrm{mg} / \mathrm{kg}$ DEX, (D) LPS + 10 mg/kg PA; (E) LPS + $20 \mathrm{mg} / \mathrm{kg} \mathrm{PA}$; (F) LPS + $40 \mathrm{mg} / \mathrm{kg}$ PA (magnification, 100x; $\mathrm{n}=6$ ).

A

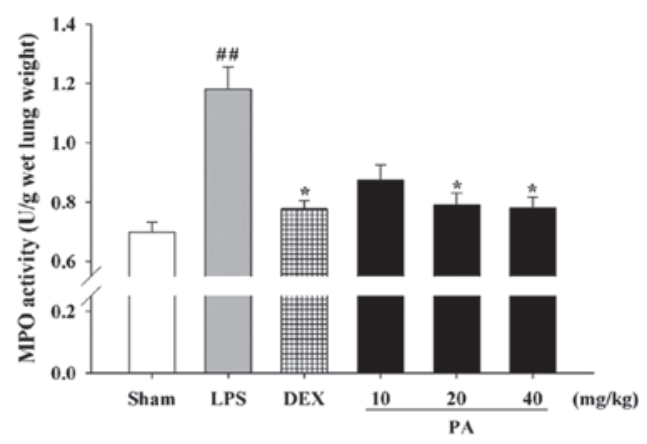

C

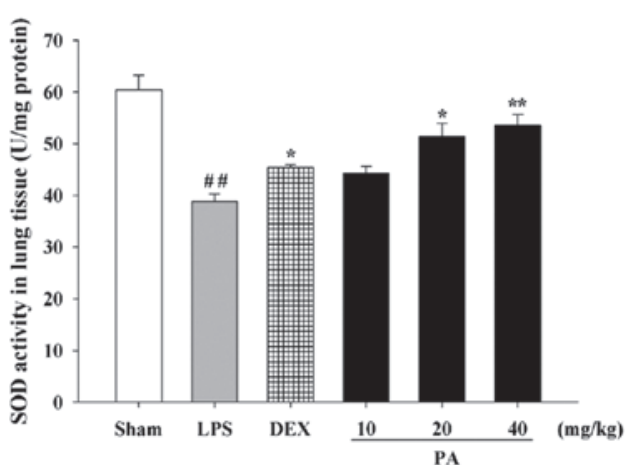

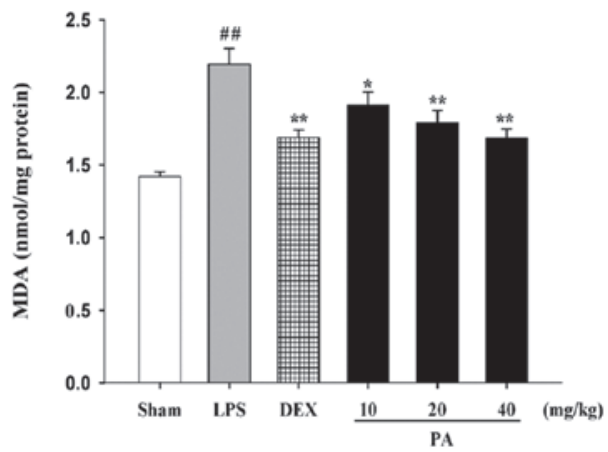

D

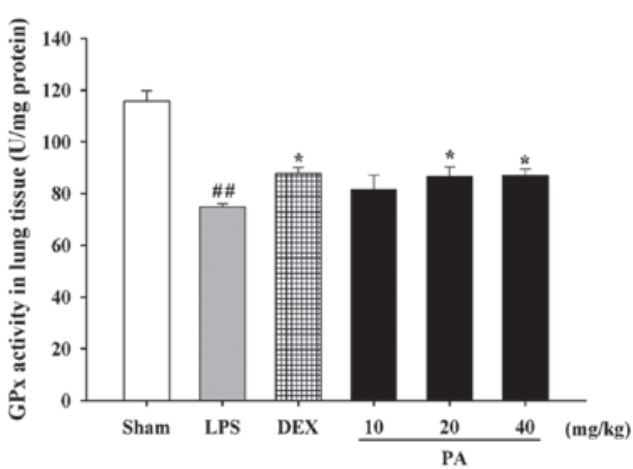

Figure 5. Effects of patchouli alcohol (PA) on myeloperoxidase (MPO), malondialdehyde (MDA), superoxide dismutase (SOD) and glutathione peroxidase (GPx) levels in the lung tissues. Mice were administered 10, 20 and $40 \mathrm{mg} / \mathrm{kg}$ PA, $5 \mathrm{mg} / \mathrm{kg}$ DEX or an equal volume of vehicle. Mice from the LPS, PA and DEX groups were administered $5 \mathrm{mg} / \mathrm{kg}$ LPS, whereas mice in the sham group received an equal volume of phosphate-buffered saline. Following $24 \mathrm{~h}$, the mice were sacrificed by cervical dislocation and the lung tissues were harvested. (A) MPO activity, (B) MDA levels (C) SOD activity and (D) GPx levels in lung tissue. Data are presented as the mean \pm standard error of the mean $(n=8) .{ }^{\# /} \mathrm{P}<0.01$ vs. the sham group; ${ }^{*} \mathrm{P}<0.05$ or ${ }^{* *} \mathrm{P}<0.01$ vs. the LPS group.

tion in the BALF was determined, which indicates endothelial permeability and pulmonary edema $(38,39)$. As hypothesized, intratracheal instillation of LPS induced a significant increase in BALF protein levels. Conversely, pretreatment with PA reduced the total protein content in the BALF. These results indicated that PA may prevent the leakage of protein-rich 

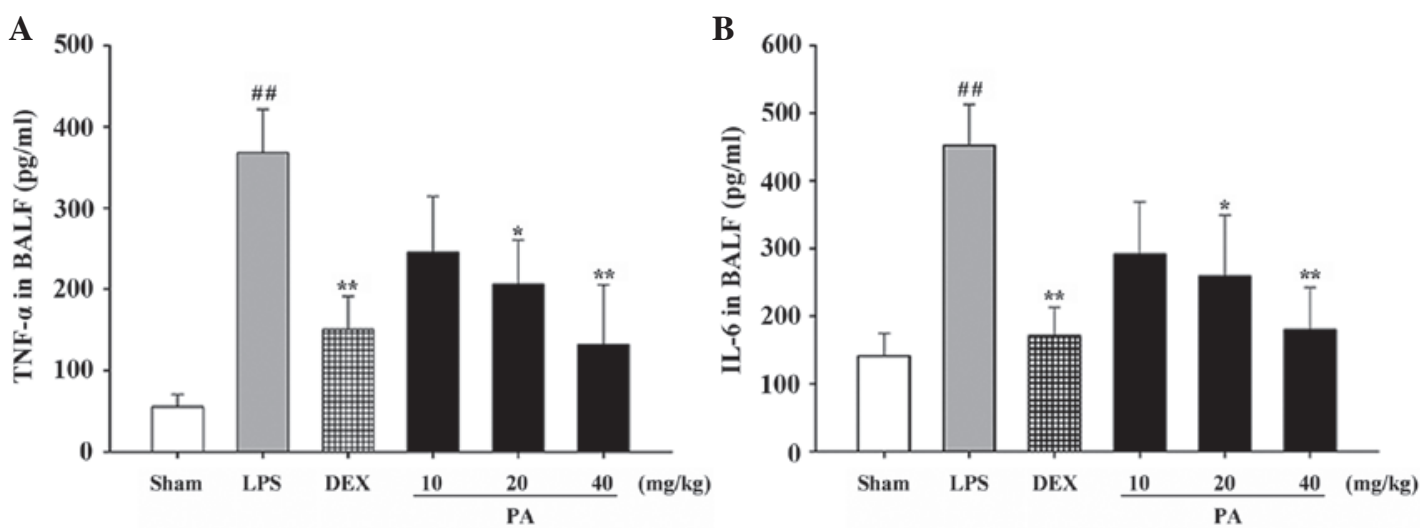

Figure 6. Effects of patchouli alcohol (PA) on cytokine production in the bronchoalveolar lavage fluid (BALF) of mice challenged with lipopolysaccharide (LPS). Mice were administered 10, 20 and $40 \mathrm{mg} / \mathrm{kg}$ PA, $5 \mathrm{mg} / \mathrm{kg}$ dexamethasone (DEX) or an equal volume of vehicle. Mice from the LPS, PA and DEX groups were administered $5 \mathrm{mg} / \mathrm{kg}$ LPS, whereas mice from sham group was administered an equal volume of phosphate-buffered saline. Following $24 \mathrm{~h}$, the mice were sacrificed by cervical dislocation and the BALF was collected. (A) Expression levels of tumor necrosis factor (TNF)- $\alpha$ in BALF. (B) Expression levels of interleukin (IL)- 6 in BALF. Data are presented as the mean \pm standard error of the mean $(n=8) .{ }^{\# \#} \mathrm{P}<0.01$ vs. the sham group; ${ }^{*} \mathrm{P}<0.05$ or ${ }^{* * *} \mathrm{P}<0.01$ vs. the LPS group.

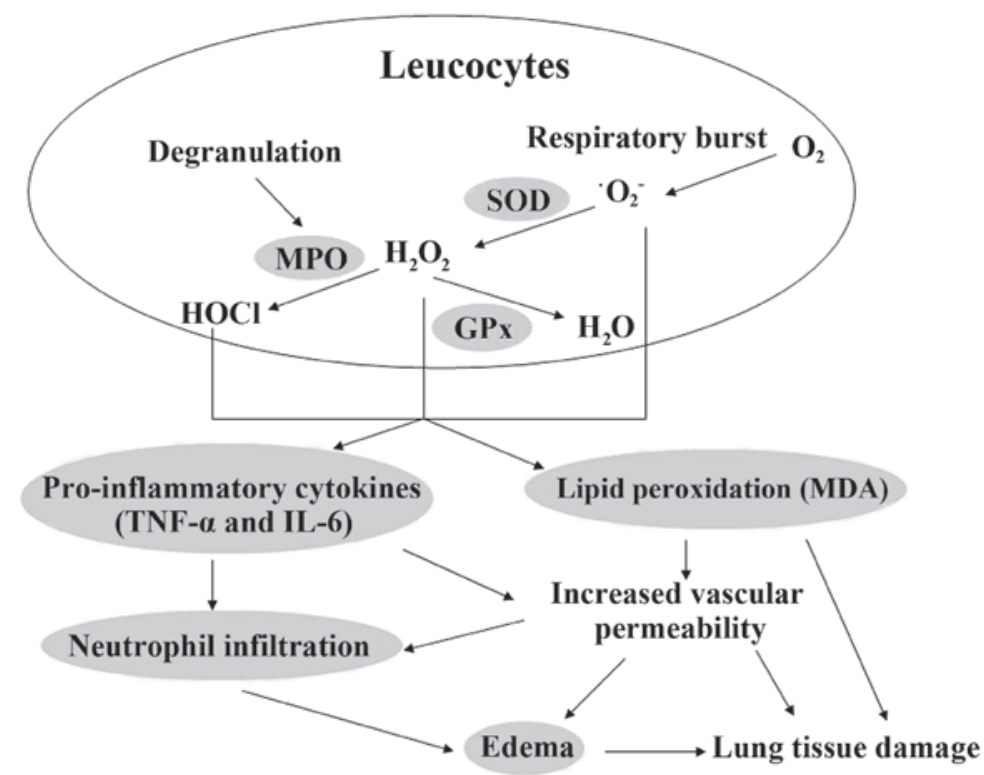

Figure 7. Schematic representation of the mechanisms underlying the protective effects induced by patchouli alcohol (PA) on lipopolysaccharide-induced acute lung injury. The shaded parts indicate the molecules/processes affected by PA. SOD, superoxide dismutase; MPO, myeloperoxidase; GPx, glutathione peroxidase; TNF, tumor necrosis factor; IL, interleukin; MDA, malondialdehyde.

fluid into the lung tissue, thus attenuating the development of pulmonary edema. Furthermore, histopathologic analysis $24 \mathrm{~h}$ following LPS challenge demonstrated significant infiltration of inflammatory cells, extensive thickening of the alveolar wall, demolished structure of pulmonary alveoli, and hemorrhage. PA pretreatment may attenuate these LPS-induced pathological changes in the lung.

MPO is an enzyme predominantly stored in the primary granules of neutrophils, therefore MPO activity in the parenchyma reflects neutrophil adhesion and margination in the lungs (40). Predominantly released by activated neutrophils, MPO is characterized by powerful pro-oxidative and proinflammatory properties (41). In addition to supporting the host defense mechanisms against infective microbes, MPO also contributes to the initiation and propagation of acute and chronic inflammatory reactions (42). However, when released, MPO may catalyze hydrogen peroxide and chloride anions to form hypochlorous acid, which may lead to tissue injury (28). Notably, LPS-induced ALI is characterized by the infiltration of neutrophils into the lung, resulting in increased MPO activity levels $(43,44)$. In the present study, pretreatment with PA significantly decreased the activity of MPO, which was consistent with reduced neutrophil infiltration in the lung tissue, thus suggesting that PA may exert anti-neutrophil influx effects in LPS-induced ALI.

Previous experimental and clinical studies have demonstrated that LPS-induced ALI may lead to a rapid overproduction of proinflammatory cytokines, including TNF- $\alpha$ and IL-6, which are characteristic cytokines associated with the inflammatory process of ALI (45-47). TNF- $\alpha$, 
which is predominantly produced by activated monocytes/macrophages, is capable of amplifying the inflammatory cascade, which may damage vascular endothelial cells (48). Furthermore, TNF- $\alpha$ is capable of inducing the production of other inflammatory cytokines, including IL-6, which stimulates the migration and adherence of neutrophils to endothelial cells (49). IL-6 is a principal cytokine mediator of the acute phase response, and a previous study suggested that IL-6 may act as a marker for predicting the severity of ALI (50). PA was previously reported to suppress the inflammatory response by inhibiting the production of proinflammatory cytokines, including TNF- $\alpha$ and IL-6 $(20,24)$. The present study demonstrated that the expression levels of TNF- $\alpha$ and IL- 6 were increased following LPS challenge, whereas pretreatment with PA significantly decreased the production of TNF- $\alpha$ and IL-6 in the BALF. These results suggested that the protective effects of PA on ALI may, at least in part, be attributed to the inhibition of inflammatory factors.

Another possible mechanism for the anti-inflammatory effects induced by PA may be associated with its antioxidant activity. Inflammatory stimuli promote the generation of ROS, generated by activated inflammatory cells and circulating enzymatic generators, which contribute to lung pathophysiology (51). When cellular production of ROS overwhelms antioxidant capacity, cellular macromolecules, including lipids, proteins and DNA, may be damaged (52). It has previously been suggested that such a state of 'oxidative stress' may contribute to the pathogenesis of numerous human diseases, including those of the lung (52). MDA is the breakdown product of polyunsaturated fatty acids following oxidation in the chain reaction of lipid peroxidation; therefore, the levels of MDA are often used as an index of oxidative stress $(53,54)$. The increase in MDA caused by lipid peroxidation may lead to the destruction of biological membranes (55). In the present study, pretreatment with PA significantly inhibited the production of MDA in a dose-dependent manner, which alleviated oxidative stress in the lung tissues of mice with ALI. Furthermore, tissues may escape ROS-induced toxic damage via ROS scavenging enzymes, including SOD and GPx, which are the first-line cellular defense against oxidative injury $(17,56)$. The equilibrium between these enzymes and ROS is important for the effective removal of oxidative stress from intracellular organelles (57). Superoxide anions are converted to hydrogen peroxide by SOD, which is then metabolized to water by GPx (28). In the present study, PA administration significantly increased the activities of SOD and GPx in the lungs, as compared with the LPS group; SOD and GPx activities were markedly reduced following LPS administration. Conversely, MDA levels were significantly decreased; therefore, the results of the present study suggested that the suppression of MDA production was associated with the increase in SOD and GPx activities. Collectively, these findings suggested that PA may effectively reduce the effects of oxidative stress in ALI.

It has previously been reported that PA has a short elimination half-life $\left(\mathrm{t}_{1 / 2 \beta}\right)$, with values of $21.51 \pm 8.46(10 \mathrm{mg} / \mathrm{kg}$, i.g. $)$, $17.81 \pm 7.52(30 \mathrm{mg} / \mathrm{kg}$, i.g. $)$ and $17.82 \pm 9.29 \mathrm{~h}(100 \mathrm{mg} / \mathrm{kg}$, i.g. $)$ detected in rats (58). PA is metabolized in the liver and kidney, and two hydroxylated metabolites have been demonstrated in the liver of rabbits (59), and one carboxylate metabolite of PA has been identified in rat urine (60). The present study demonstrated that intragastric administration of PA has a potent protective effect against LPS-induced ALI in mice. The mechanism may be related to the anti-inflammatory and antioxidative activities of PA and/or its metabolites. Although our previous in vitro study demonstrated that PA has direct anti-inflammatory activity in RAW264.7 macrophages (24), further investigation is required to clarify this.

In conclusion, the results of the present study demonstrated that pretreatment with PA improved the survival rate of mice with LPS-induced ALI, and effectively attenuated LPS-induced ALI by inhibiting pulmonary histopathologic alterations. The mechanisms underlying this protective effect included (i) reduced W/D ratio and protein leakage; (ii) reduced MPO activity levels; (iii) reduced lipid peroxidation and MDA formation; (iv) elevated activity of antioxidative enzymes, including SOD and GPx; and (v) decreased secretion of proinflammatory cytokines, including TNF- $\alpha$ and IL-6 (Fig. 7). These results suggested that PA may be a potential therapeutic agent for the prevention of ALI.

\section{Acknowledgements}

The present study was supported by grants from: The National Natural Science Foundation of China (grant nos. 81303200 and 81403169); the Natural Science Foundation of Guangdong Province (grant no. S2013010016627); the Administration of Traditional Chinese Medicine Project of Guangdong Province (grant no. 20132142); the Medical Scientific Research Foundation of Guangdong Province (grant no. A2013232); the Combined Program of Ministry of Education of Guangdong Province (grant no. 2012B090600007); the Science and Technology Cooperation Project of Hong Kong, Macao, and Taiwan (grant no. 2014DFH30010); and the Special Funds from Central Finance of China in Support of the Development of Local Colleges and University [grant no. 276 (2014)].

\section{References}

1. Yuan X, Wang Y, Du D, Hu Z, Xu M, Xu M and Liu Z: The effects of the combination of sodium ferulate and oxymatrine on lipopolysaccharide-induced acute lung injury in mice. Inflammation 35: 1161-1168, 2012.

2. Wu WS, Chou MT, Chao CM, Chang CK, Lin MT and Chang CP: Melatonin reduces acute lung inflammation, edema and hemorrhage in heatstroke rats. Acta Pharmacol Sin 33: 775-782, 2012.

3. Matsuyama H, Amaya F, Hashimoto S, Ueno H, Beppu S, Mizuta M, Shime N, Ishizaka A and Hashimoto S: Acute lung inflammation and ventilator-induced lung injury caused by ATP via the P2Y receptors: an experimental study. Respir Res 9: 79, 2008.

4. Wyncoll DL and Evans TW: Acute respiratory distress syndrome. Lancet 354: 497-501, 1999.

5. Abraham E: Neutrophils and acute lung injury. Critical Care Medicine 31: S195-S199, 2003.

6. Ware LB and Matthay MA: The acute respiratory distress syndrome. N Engl J Med 342: 1334-1349, 2000.

7. Atabai K and Matthay MA: The pulmonary physician in critical care. 5: Acute lung injury and the acute respiratory distress syndrome: Definitions and epidemiology. Thorax 57: 452-458, 2002.

8. Rubenfeld GD, Caldwell E, Peabody E, Weaver J, Diane P, Martin DP, Neff M, Stern EJ and Hudson LD: Incidence and outcomes of acute lung injury. N Engl J Med 353: 1685-1693, 2005.

9. Knight PR, Druskovich G, Tait AR and Johnson KJ: The role of neutrophils, oxidants and proteases in the pathogenesis of acid pulmonary injury. Anesthesiology 77: 772-778, 1992. 
10. Yunhe F, Bo L, Xiaosheng F, Fengyang L, Dejie L, Zhicheng L, Depeng L, Yongguo C, Xichen Z, Naisheng Z and Zhengtao Y: The effect of magnolol on the Toll-like receptor 4/nuclear factor kappaB signaling pathway in lipopolysaccharide-induced acute lung injury in mice. Eur J Pharmacol 689: 255-261, 2012.

11. Szarka RJ, Wang N, Gordon L, Nation PN and Smith RH: A murine model of pulmonary damage induced by lipopolysaccharide via intranasal instillation. J Immunol Methods 202: 49-57, 1997.

12. Ulich TR, Yin S, Remick DG, Russell D, Eisenberg SP and Kohno T: Intratracheal administration of endotoxin and cytokines IV. The soluble tumor necrosis factor receptor type I inhibits acute inflammation. Am J Pathol 142: 1335-1338, 1993.

13. Zhang X, Huang H, Yang T, Ye Y, Shan J, Yin Z and Luo L: Chlorogenic acid protects mice against lipopolysaccharide-induced acute lung injury. Injury 41: 746-752, 2010.

14. Sibille Y and Reynolds HY: Macrophages and polymorphonuclear neutrophils in lung defense and injury. Am Rev Respir Dis 141: 471-501, 1990

15. Matthay MA and Zimmerman GA: Acute lung injury and the acute respiratory distress syndrome: Four decades of inquiry into pathogenesis and rational management. Am J Respir Cell Mol Biol 33: 319-327, 2005.

16. Edem VF, Kosoko A, Akinyoola SB, Owoeye O, Rahamon SK and Arinola OG: Plasma antioxidant enzymes, lipid peroxidation and hydrogen peroxide in wistar rats exposed to Dichlorvos insecticide. Archives of Applied Science Research 4: 1778-1781 2012.

17. Huang $\mathrm{CH}$, Yang ML, Tsai CH, Li YC, Lin YJ and Kuan YH: Ginkgo biloba leaves extract (EGb 761) attenuates lipopolysaccharide-induced acute lung injury via inhibition of oxidative stress and NF-kappaB-dependent matrix metalloproteinase- 9 pathway. Phytomedicine 20: 303-309, 2013.

18. Li YC, Peng SZ, Chen HM, Zhang FX, Xu PP, Xie JH, He JJ, Chen JN, Lai XP and Su ZR: Oral administration of patchouli alcohol isolated from Pogostemonis Herba augments protection against influenza viral infection in mice. Int Immunopharmacol 12: 294-301, 2012.

19. Li CW, Wu XL, Zhao XN, Su ZQ, Chen HM, Wang XF, Zhang XJ, Zeng HF, Chen JN, Li YC and Su ZR: Anti-inflammatory property of the ethanol extract of the root and rhizome of Pogostemon cablin (Blanco) Benth. ScientificWorldJournal 2013: Article ID 434151, 2013.

20. Li YC, Xian YF, Ip SP, Su ZR, Su JY, He JJ, Xie QF, Lai XP and Lin ZX: Anti-inflammatory activity of patchouli alcohol isolated from Pogostemonis Herba in animal models. Fitoterapia 82: 1295-1301, 2011.

21. Kim HW, Cho SJ, Kim BY, Cho SI and Kim YK: Pogostemon cablin as ROS Scavenger in Oxidant-induced Cell death of human neuroglioma cells. Evid Based Complement Alternat Med 7: 239-247, 2010.

22. Liu X, Fan R, Zhang Y and Zhu M: Study on antimicrobial activities of extracts from Pogostemon cablin (Blanco) Benth. Food Sci Technol 34: 220-227, 2009

23. Lu TC, Liao JC, Huang TH, Lin YC, Liu CY, Chiu YJ and Peng WH: Analgesic and anti-inflammatory activities of the methanol extract from Pogostemon cablin. Evid Based Complement Alternat Med 2011: 671741, 2011.

24. Xian YF, Li YC, Ip SP, Lin ZX, Lai XP and Su ZR: Anti-inflammatory effect of patchouli alcohol isolated from Pogostemonis Herba in LPS-stimulated RAW264.7 macrophages. Exp Ther Med 2: 545-550, 2011.

25. Huang XW, Bai L, Xu FH and Wu YJ: Inhibitory activities of patchouli alcohol on neurotoxicity of $\beta$-amyloid peptide. Jie Fang Jun Yi Xue Za Zhi 24: 338-340, 2008

26. Liao JB, Wu DW, Peng SZ, Xie JH, Li YC, Su JY, Chen JN and Su ZR: Immunomodulatory Potential of patchouli alcohol isolated from Pogostemon cablin (Blanco) Benth (Lamiaceae) in mice. Tropical Journal of Pharmaceutical Research 12: 559-565, 2013.

27. Mehla K, Balwani S, Agrawal A and Ghosh B: Ethyl gallate attenuates acute lung injury through Nrf2 signaling. Biochimie 95 2404-2414, 2013.

28. Yeh CH, Yang JJ, Yang ML, Li YC and Kuan YH: Rutin decreases lipopolysaccharide-induced acute lung injury via inhibition of oxidative stress and the MAPK-NF- $\mathrm{KB}$ pathway. Free Radic Biol Med 69: 249-257, 2014

29. Bai GZ, Yu HT, Ni YF, Li XF, Zhang ZP, Su K, Lei J, Liu BY, Ke CK, Zhong DX, et al: Shikonin attenuates lipopolysaccharide-induced acute lung injury in mice. J Surg Res 182: 303-311, 2013
30. Zhong WT, Jiang LX, Wei JY, Qiao AN, Wei MM, Soromou LW, Xie XX, Zhou X, Ci XX and Wang DC: Protective effect of esculentoside A on lipopolysaccharide-induced acute lung injury in mice. J Surg Res 185: 364-372, 2013.

31. Sun Q, Chen L, Gao M, Jiang W, Shao F, Li J, Wang J, Kou J and Yu B: Ruscogenin inhibits lipopolysaccharide-induced acute lung injury in mice: involvement of tissue factor, inducible $\mathrm{NO}$ synthase and nuclear factor (NF)-kappaB. Int Immunopharmacol 12: 88-93, 2012.

32. Gao M, Chen L, Yu H, Sun Q, Kou J and Yu B: Diosgenin down-regulates NF-kappaB p65/p50 and p38MAPK pathways and attenuates acute lung injury induced by lipopolysaccharide in mice. Int Immunopharmacol 15: 240-245, 2013.

33. Liang XM, Guo GF, Huang XH, Duan WL and Zeng ZL: Isotetrandrine protects against lipopolysaccharide-induced acute lung injury by suppression of mitogen-activated protein kinase and nuclear factor-kappa B. J Surg Res 187: 596-604, 2014.

34. Xu GL, Yao L, Rao SY, Gong ZN, Zhang SQ and Yu SQ: Attenuation of acute lung injury in mice by oxymatrine is associated with inhibition of phosphorylated p38 mitogen-activated protein kinase. J Ethnopharmacol 98: 177-183, 2005.

35. Zhang B, Liu ZY, Li YY, Luo Y, Liu ML, Dong HY, Wang YX, Liu Y, Zhao PT, Jin FG and Li ZC: Antiinflammatory effects of matrine in LPS-induced acute lung injury in mice. Eur J Pharm Sci 44: 573-579, 2011.

36. Faffe DS, Seidl VR, Chagas PS, Gonçalves de Moraes VL, Capelozzi VL, Rocco PR and Zin WA: Respiratory effects of lipopolysaccharide-induced inflammatory lung injury in mice. Eur Respir J 15: 85-91, 2000

37. Perina DG: Noncardiogenic pulmonary edema. Emerg Med Clin North Am 21: 385-393, 2003

38. Beck BD, Brain JD and Bohannon DE: An in vivo hamster bioassay to assess the toxicity of particulates for the lungs. Toxicol Appl Pharmacol 66: 9-29, 1982.

39. Zhang X, Song K, Xiong H, Li H, Chu X and Deng X: Protective effect of florfenicol on acute lung injury induced by lipopolysaccharide in mice. Int Immunopharmacol 9: 1525-1529, 2009.

40. Klebanoff SJ: Myeloperoxidase: friend and foe. J Leukoc Biol 77: 598-625, 2005

41. Loria V, Dato I, Graziani F and Biasucci LM: Myeloperoxidase: A new biomarker of inflammation in ischemic heart disease and acute coronary syndromes. Mediators Inflamm 2008: 135625, 2008.

42. Alfakry H, Sinisalo J, Paju S, Nieminen MS, Valtonen V, Tervahartiala T, Pussinen PJ and Sorsa T: The association of serum neutrophil markers and acute coronary syndrome. Scand J Immunol 76: 181-187, 2012.

43. Moraes TJ, Zurawska JH and Downey GP: Neutrophil granule contents in the pathogenesis of lung injury. Curr Opin Hematol 13: 21-27, 2006

44. Yao HY, Zhang LH, Shen J, Shen HJ, Jia YL, Yan XF and Xie QM: Cyptoporus polysaccharide prevents lipopolysaccharide-induced acute lung injury associated with down-regulating Toll-like receptor 2 expression. J Ethnopharmacol 137: 1267-1274, 2011.

45. Bhatia M and Moochhala S: Role of inflammatory mediators in the pathophysiology of acute respiratory distress syndrome. J Pathol 202: 145-156, 2004.

46. Goodman RB, Pugin J, Lee JS and Matthay MA: Cytokine-mediated inflammation in acute lung injury. Cytokine Growth Factor Rev 14: 523-535, 2003.

47. Cribbs SK, Matthay MA and Martin GS: Stem cells in sepsis and acute lung injury. Crit Care Med 38: 2379-2385, 2010.

48. Giebelen IA, van Westerloo DJ, LaRosa GJ, de Vos AF and van der Poll T: Local stimulation of alpha7 cholinergic receptors inhibits LPS-induced TNF-alpha release in the mouse lung. Shock 28: 700-703, 2007.

49. Liang X, Wang RS, Wang F, Liu S, Guo F, Sun L, Wang YJ, Sun YX and Chen XL: Sodium butyrate protects against severe burn-induced remote acute lung injury in rats. PLoS One 8: e68786, 2013

50. Leser HG, Gross V, Scheibenbogen C, Heinisch A, Salm R, Lausen M, Rückauer K, Andreesen R, Farthmann EH and Schölmerich J: Elevation of serum interleukin- 6 concentration precedes acute-phase response and reflects severity in acute pancreatitis. Gastroenterology 101: 782-785, 1991

51. Gow AJ, Farkouh CR, Munson DA, Posencheg MA and Ischiropoulos H: Biological significance of nitric oxide-mediated protein modifications. Am J Physiol Lung Cell Mol Physiol 287: L262-268, 2004 
52. Thannickal VJ and Fanburg BL: Reactive oxygen species in cell signaling. Am J Physiol Lung Cell Mol Physiol 279: L1005-1028, 2000.

53. Chevalier G, Ricard AC and Manca D: Age-related variations of lipid peroxidation in cadmium-treated rats. Toxicol Ind Health 10: 43-51, 1994.

54. Manca D, Ricard AC, Trottier B and Chevalier G: Studies on lipid peroxidation in rat tissues following administration of low and moderate doses of cadmium chloride. Toxicology 67: 303-323, 1991.

55. Draper $\mathrm{H}$ and Hadley M: Malondialdehyde determination as index of lipid peroxidation. Methods Enzymol 186: 421-431, 1990.

56. Vijayaraj P, Muthukumar K, Sabarirajan J and Nachiappan V: Antihyperlipidemic activity of Cassia auriculata flowers in triton WR 1339 induced hyperlipidemic rats. Exp Toxicol Pathol 65: 135-141, 2013.
57. Kareem MA, Gadhamsetty SK, Shaik AH, Prasad EM and Kodidhela LD: Protective effect of nutmeg aqueous extract against experimentally-induced hepatotoxicity and oxidative stress in rats. J Ayurveda Integr Med 4: 216-223, 2013.

58. Zhang R, Yan P, Li Y, Xiong L, Gong X and Peng C: A pharmacokinetic study of patchouli alcohol after a single oral administration of patchouli alcohol or patchouli oil in rats. Eur J Drug Metab Pharmacokinet: March 10, 2015 (Epub ahead of print).

59. Bang L, Ourisson G and Teisseire P: Hydroxylation of patchoulol by rabbits. Hemi-synthesis of nor-patchoulenol, the odour carrier of patchouli oil. Tetrahedron Letters 26: 2211-2214, 1975.

60. Bang L, Ourisson G and Teisseire P. Hydroxylation of patchoulol by rabbits. Hemi-synthesis of nor-patchoulenol, the odour carrier of patchouli Oil. Tetrahedron Letters 16: 2211-2214, 1975. 